compared outcomes before the introduction of HiP in Scotland and immediately after its withdrawal with those during the period for which it existed, and by varying the timing of the intervention period, ascertain if observed effects could be attributed to $\mathrm{HiP}$ or other co-existing policies.

Methods All singleton births with a gestational age 26-44 weeks were extracted from routinely collected birth data between January 2004 and December 2013. The outcomes of interest were birthweight and contacting midwife before 25 weeks. Interrupted time series models, adjusting for maternal, birth characteristics, seasonality and temporal trends, were used. The intervention period (April 2009 to April 2011) and post-intervention period (May 2011 to December 2013) were compared to the pre-intervention period (January 2004 to March 2009). Four different start dates (6 \& 12 months before and after April 2009) and three duration periods (18, 24, 30 months) were chosen.

Results There were 272,935 births pre-intervention, 109,775 during the intervention and 144,663 post-intervention. Compared to pre-intervention, birthweight remained unchanged in both the intervention period $-2.6 \mathrm{~g}(95 \% \mathrm{CI}-6.9 ; 1.7)$ and post-intervention $-5.0 \mathrm{~g}(-11.1 ; 1.0)$. During the intervention period women were more likely to contact the midwife OR $1.1095 \%$ CI $(1.02 ; 1.18)$. For birthweight, the duration of the intervention did not alter the effect of HiP. The effect on contacting a midwife was similar across start dates and duration periods.

Conclusion The strongest intervention effects did not coincide with the dates during which the HiP grant was in place, and there was little dilution of the effects, meaning any effects found could be due to policies and events other than the HiP grant. The lack of random allocation to intervention means the groups were exposed to different macro-level environments, which may impact on birth outcomes during the intervention period.

\section{OP85 PATIENT AND STAFF PERCEPTIONS OF SAFETY AND RISK: TRIANGULATING PATIENT COMPLAINTS AND STAFF INCIDENT REPORTS TOWARDS A DUAL PERSPECTIVE ON ADVERSE EVENTS}

\footnotetext{
'J van Dael ${ }^{*},{ }^{2}$ AT Gillespie, ${ }^{2}$ TW Reader, ${ }^{1}$ EK Mayer. 'Institute of Global Health Innovation, Imperial College, London, UK; ${ }^{2}$ Department of Psychology and Behavioural Sciences, London School of Economics, London, UK
}

\subsection{6/jech-2020-SSMabstracts.84}

Background Incident reporting systems in healthcare are historically based on staff descriptions of adverse events. An increasing body of literature suggests patients provide critical insights to risk and error, but their potential has not sufficiently been investigated at the incident level. This study aims to examine to what extent patient complaints and staff incident reports discuss identical incidents, and how their perspectives could be integrated for more comprehensive safety analysis.

Methods Deterministic data linkage was performed on all complaints $(n=5,265)$ and staff incident reports ('PSIs') $(n=81,077)$ between April 2014 and March 2019 at a multisite hospital in London. A total of 402 complaints covered at least one incident also identified in the PSIs, and were included in the study. All incidents reported in complaints and staff incident reports were codified based on problem domain; problem severity; stage of care; staff group implicated; reported harm; and descriptive level (eg, description of human factors and root causes); adapted from the Healthcare Complaints Analysis Tool (HCAT) and the National Reporting and Learning System (NRLS). Aggregated coding outputs informed targeted qualitative analysis of free text incident reports for an in-depth exploration of key overlap and discrepancies in patient and staff descriptions of unsafe care.

Results Our preliminary results indicate staff and patients reported similar problem themes for $81.1 \%$ of overlapping incidents (of which $66.5 \%$ clinical, followed by $27.1 \%$ institutional, and $6.4 \%$ relational), but commonly differed in their description of contributing factors and root causes (eg, different time points in patient journey). Alongside overlapping incidents, patients reported an average of 1.4 additional incidents in their complaint, of which $23.6 \%$ were high severity. Additional patient-reported incidents included blind spot clinical issues $(36.7 \%$; eg care continuity; care omissions) or relationship issues pre- or post mutually identified incidents $(39.1 \%$; eg failure to listen to patient concerns; breach of candour).

Conclusion Our study suggests that traditional, clinician-based models of safety and risk are likely to omit critical dimensions of root causes to adverse events. Patients and public are able to contribute to safety monitoring and evaluation in two ways: by highlighting overlooked or undervalued aspects of unsafe care (eg failure to listen; absent communication) and by revealing latent causes of incidents across time (eg failed continuity of care; systemic care omissions).

\section{OP86 LOW ECONOMIC GROWTH, HEALTH, HEALTH INEQUALITIES AND SUSTAINABLE DEVELOPMENT GOALS IN A RICH COUNTRY: 27-YEAR JAPANESE TIME SERIES}

\begin{abstract}
${ }^{1,2,3}$ A Hiyoshi ${ }^{*},{ }^{4} \mathrm{~K}$ Honjo, ${ }^{5} \mathrm{LG}$ Platts, ${ }^{4} \mathrm{Y}$ Suzuki, ${ }^{3} \mathrm{MJ}$ Shipley, ${ }^{6} \mathrm{H}$ Iso, ${ }^{7} \mathrm{~N}$ Kondo, ${ }^{3}$ EJ Brunner. ${ }^{1}$ Clinical Epidemiology and Biostatistics, School of Medical Sciences, Orebro University, Orebro, Sweden; ${ }^{2}$ Department of Public Health Sciences, Stockholm University, Stockholm, Sweden; ${ }^{3}$ Department of Epidemiology and Public Health, University College London, London, UK; ${ }^{4}$ Social and Behavioral Sciences, Osaka Medical College, Takatsuki, Japan; ${ }^{5}$ Stress Research Institute, Department of Psychology, Stockholm University, Stockholm, Sweden; ${ }^{6}$ Public Health, Department of Social Medicine, Osaka University Graduate School of Medicine, Osaka, Japan; ${ }^{7}$ School of Public Health, University of Tokyo, Tokyo, Japan
\end{abstract}

\subsection{6/jech-2020-SSMabstracts.85}

Background Sustainable Development Goal \#8 refers to decent work and economic growth. In the context of climate change and global resource depletion, it is important to understand whether low economic growth is compatible with positive population health in rich countries, particularly because the past decade of austerity in the UK and USA is associated with stagnating life expectancy. Japan provides a natural experiment in that it has experienced low economic growth since 1992, and life expectancy continued to improve. However, the trend in health inequality in good self-rated health is unknown.

Methods We examined trends in health and health inequalities using ten triennial waves of a nationally representative survey in Japan, 1986-2013 $(\mathrm{n}=731,647)$. Change in age-standardized self-rated good health was calculated, and health inequalities and their time trends were calculated using Slope and Relative Indices of Inequality (SII and RII respectively) in relation to net household income. Analyses were stratified by sex and age, for children (6-18 years), working-age adults (20-59 years), younger old (60-69 years) and older old (7079 years), given age differences in relation to the economy 